\title{
Quantifying stoichiometry of mixed-cation-anion III-V semiconductor interfaces at atomic resolution
}

K. Mahalingam, K. G. Eyink, G. J. Brown, D. L. Dorsey, C. F. Kisielowski, and A. Thust

Citation: Appl. Phys. Lett. 88, 091904 (2006); doi: 10.1063/1.2178771

View online: https://doi.org/10.1063/1.2178771

View Table of Contents: http://aip.scitation.org/toc/apl/88/9

Published by the American Institute of Physics

\section{Articles you may be interested in}

Strain analysis of compositionally tailored interfaces in $\mathrm{InAs} / \mathrm{GaSb}$ superlattices

Applied Physics Letters 103, 211605 (2013); 10.1063/1.4833536

Atomic intermixing in short-period InAs/GaSb superlattices

Applied Physics Letters 100, 241604 (2012); 10.1063/1.4729058

Interfacial intermixing in InAs/GaSb short-period-superlattices grown by molecular beam epitaxy Applied Physics Letters 96, 021904 (2010); 10.1063/1.3291666

Evaluation of antimony segregation in $\ln A s / \ln A s_{1-x} S_{b}$ type-II superlattices grown by molecular beam epitaxy Journal of Applied Physics 119, 095702 (2016); 10.1063/1.4942844

Evaluation of thickness and strain of thin planar layers of InAs on $\mathrm{GaAs}(001)$ using spectroscopic ellipsometry Applied Physics Letters 105, 031909 (2014); 10.1063/1.4890236

Quantitative analysis of strain distribution in $\ln A s / \ln A s_{1-x} \mathrm{Sb}_{x}$ superlattices

Applied Physics Letters 103, 061908 (2013); 10.1063/1.4817969

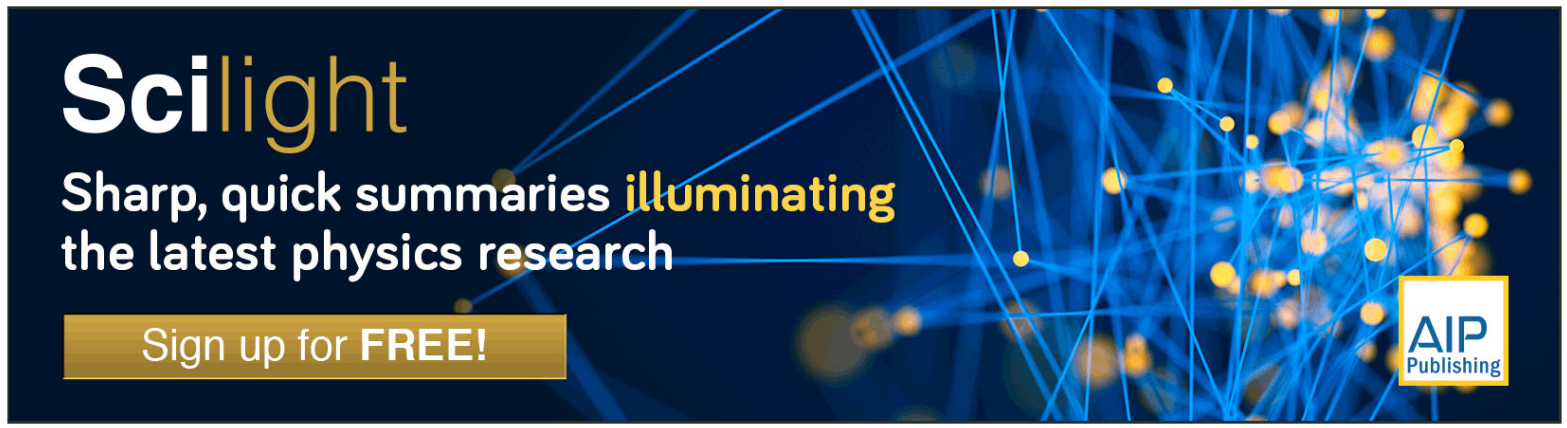




\title{
Quantifying stoichiometry of mixed-cation-anion III-V semiconductor interfaces at atomic resolution
}

\author{
K. Mahalingam, ${ }^{\text {a) }}$ K. G. Eyink, G. J. Brown, and D. L. Dorsey \\ Air Force Research Laboratory, Wright-Patterson Air Force Base, Ohio 45433 \\ C. F. Kisielowski \\ NCEM, Lawrence Berkeley National Laboratory, Berkeley, California 94720
}

A. Thust

Institut für Festkörperforschung, Forschungszentrum Jülich GmbH, D-52425 Jülich, Germany

(Received 25 October 2005; accepted 16 January 2006; published online 27 February 2006)

\begin{abstract}
Employing the focal-series reconstruction technique in high-resolution transmission electron microscopy, we obtained true atomic resolution images of interfacial disorder within cation and anion sublattices across interfaces in an InGaSb/InAs heterostructure. This enabled independent quantitative mapping of changes in the $\mathrm{In}-\mathrm{Ga}$ and $\mathrm{As}-\mathrm{Sb}$ contents across interfacial regions $\sim 0.6 \mathrm{~nm}$ in width. A comparison of the cation and anion sublattice images revealed that intermixing at the InGaSb-on-InAs interface is confined to the In-Ga sublattice. Also, atomic scale roughness within the As-Sb sublattice of the InAs-on-InGaSb interface was discerned. This approach is general, permitting atomic-scale compositional analysis of heterointerfaces with two species per sublattice. () 2006 American Institute of Physics. [DOI: 10.1063/1.2178771]
\end{abstract}

Mixed-cation-anion III-V heterostructure, derived from InAs/ $\mathrm{In}_{x} \mathrm{Ga}_{(1-x)} \mathrm{Sb}$ and InAs/AlSb systems, have attracted significant interest for a variety of applications including terahertz transistors, mid infrared lasers, and longwavelength detectors. ${ }^{1}$ The versatility of these heterostructures is based on the principle that their electronic and optical properties can be tuned by appropriately designing the thickness and composition of the individual layers. However, several studies show that stoichiometry of their interfaces strongly influences both structural and optoelectronic properties. ${ }^{2-4}$ Consequently, optimizing these properties through precise tailoring of interface composition has received much attention. ${ }^{5,6}$ A key requirement in such efforts is the capability to accurately quantify interface stoichiometry.

Heterostructures with nominally tailored interfaces have previously been investigated by high-resolution transmission electron microscopy (HRTEM), which indicated interfacial disorder on the order of 1-2 monolayers. ${ }^{4,7}$ However, chemical analysis of these interfaces was not possible because the HRTEM-based methods developed are applicable only to ternary systems (such as GaAs-AlAs), where change in composition occurs in only one sublattice. ${ }^{8-10}$ In this letter, we propose a different approach where, in place of conventional HRTEM images, the electron wave function at the exit surface of the transmission electron microscope (TEM) sample, also known as the exit-plane wave function (EPWF), ${ }^{11}$ is employed.

The use of EPWF offers important advantages over conventional HRTEM images. In addition to an improved spatial resolution, approaching the information limit of the TEM, imaging artifacts caused either by nonlinearity of the imaging process or by unwanted aberrations of the objective lens, such as spherical aberration, are eliminated. This work employs the focal-series reconstruction technique, which retrieves the EPWF from a series of HRTEM images acquired

${ }^{a)}$ Electronic mail: krishnamurthy.mahalingam@wpafb.af.mil with different settings of the objective lens defocus. ${ }^{11}$ The implicit averaging over a series of images leads to an improvement in the signal/noise ratio, which in principle is by a factor of $\sqrt{N / 2}$ for $N$ images. ${ }^{12}$ The results from the present study show that the phase of the EPWF (hereafter referred to as "phase image") clearly reveals changes in stoichiometry across interfaces at atomic resolution. The method of factorial analysis of correspondence (FAC) is used to extract the compositional profile representing the observed changes in stoichiometry. ${ }_{10,13,14}$

The proposed approach is demonstrated by analyzing an $\mathrm{In}_{0.25} \mathrm{Ga}_{0.75} \mathrm{Sb} / \mathrm{InAs}$ superlattice structure, which was grown on a (100)-GaSb substrate by molecular-beam epitaxy. Figure 1 is a (200) dark-field TEM image showing the alternating InAs and $\mathrm{In}_{0.25} \mathrm{Ga}_{0.75} \mathrm{Sb}$ layers, of nominal thickness $5.2 \mathrm{~nm}$ and $2.0 \mathrm{~nm}$, respectively. The interfacial regions are clearly distinguished by the distinct dark contrast present at each interface. HRTEM imaging of these interfaces was performed using the Philips CM300 FEG/UT TEM operated at $300 \mathrm{kV}$. Cross-sectional samples for observations were prepared by ion milling, following procedures described in a previous report. ${ }^{15}$ The experimental setup and procedures for implementing the focal-series reconstruction technique are also described in that report. ${ }^{15}$ The interfaces were imaged along the [001] zone axis to obtain adequate separation be-

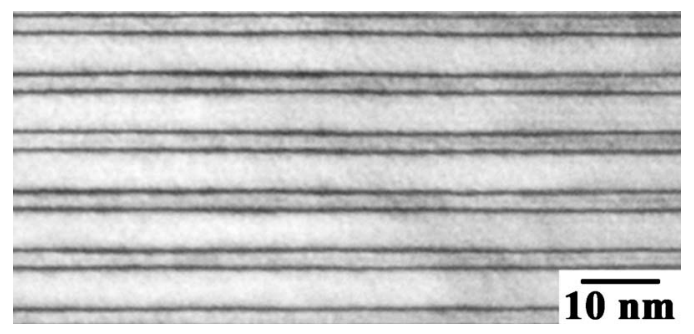

FIG. 1. A (200) dark-field TEM image of an InAs/InGaSb superlattice, showing the InAs (bright) and InGaSb (mid-grey) layers. Note the presence of dark contrast at the interfacial regions. 

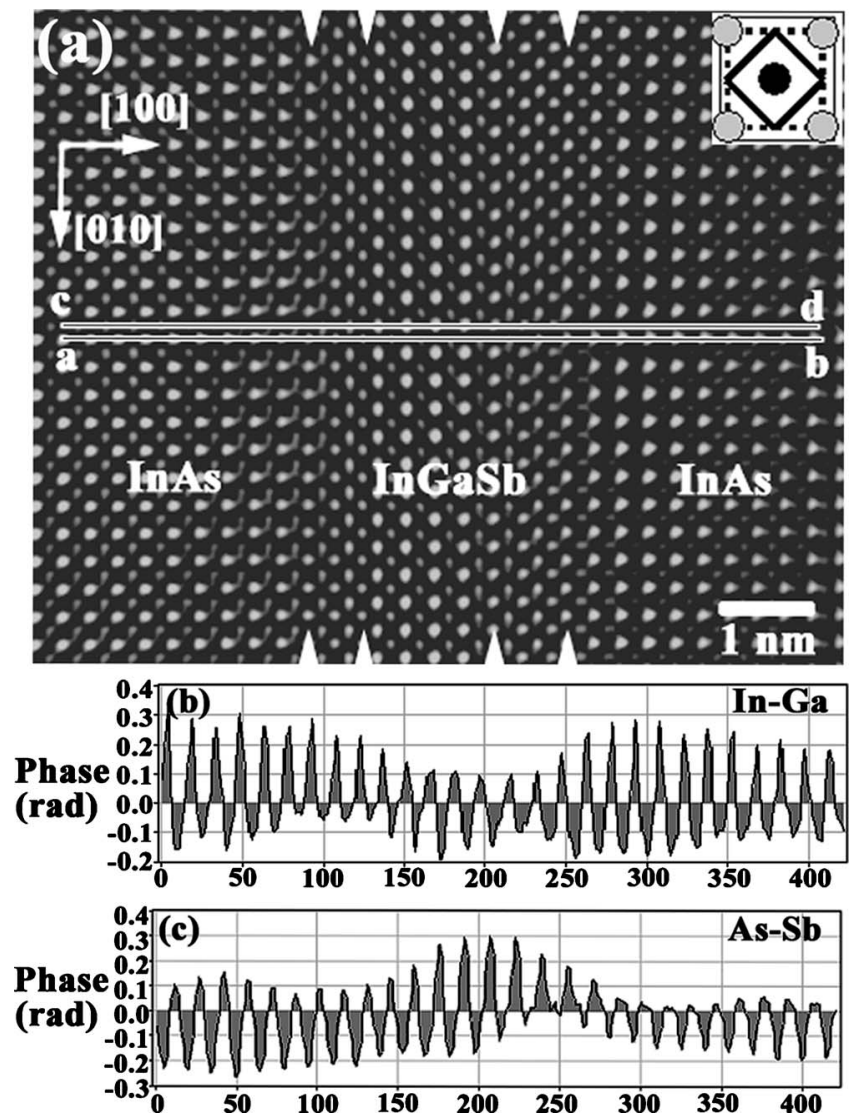

FIG. 2. (a) EPWF-phase image of the InAs/InGaSb structure, with arrows locating the interfacial regions; (b) and (c) are, respectively, the line profiles along the In-Ga (line a-b), and adjacent As-Sb (line c-d) sublattices. The inset in (a) is the projected zinc-blende unit cell. The solid lines define the subcell centered on each atomic site $\left(0.2 \times 0.2 \mathrm{~nm}^{2}\right)$, which was analyzed using the FAC procedure.

tween the cation and anion sublattices. Based on dynamical diffraction calculations, the thickness of the regions examined is estimated to be in the range of $4 \mathrm{~nm}-6 \mathrm{~nm}$. The numerical reconstruction procedure and subsequent correction of residual aberrations were performed using the TrueImage software (FEI Co., Hillsboro, OR). Prior to performing the reconstruction, a noise reduction procedure employing the background subtraction filter ${ }^{16}$ was applied to the HRTEM images to reduce contributions from the amorphous layers on the top and bottom surfaces of the sample.

The EPWF obtained by reconstruction was decomposed into amplitude and phase components. Based on the phase object approximation, for the range of specimen thickness examined in this study, the projected atomic structure is revealed in the phase image, where the atomic columns exhibit a local phase maxima (bright dots), with values proportional to their respective projected potentials. Figure 2(a) is a phase image of an InAs/InGaSb/InAs structure in Fig. 1 wherein, the atomic sites with stronger (weaker) phase maxima correspond to the In (As) sublattice within InAs layer, and the $\mathrm{Sb}$ (In-Ga) sublattice within InGaSb layer. The arrows in Fig. 2(a) denote the interfacial regions corresponding to those exhibiting dark contrast in Fig. 1, indicating that these regions are about four atomic layers $(\sim 0.6 \mathrm{~nm})$ in width. A close inspection of the atomic sites within these regions reveals a systematic change in their respective phase maxima. This is easily recognized in the line profiles across interfaces along adjacent $\mathrm{In}-\mathrm{Ga}$ and $\mathrm{As}-\mathrm{Sb}$ sublattices, shown in Figs.
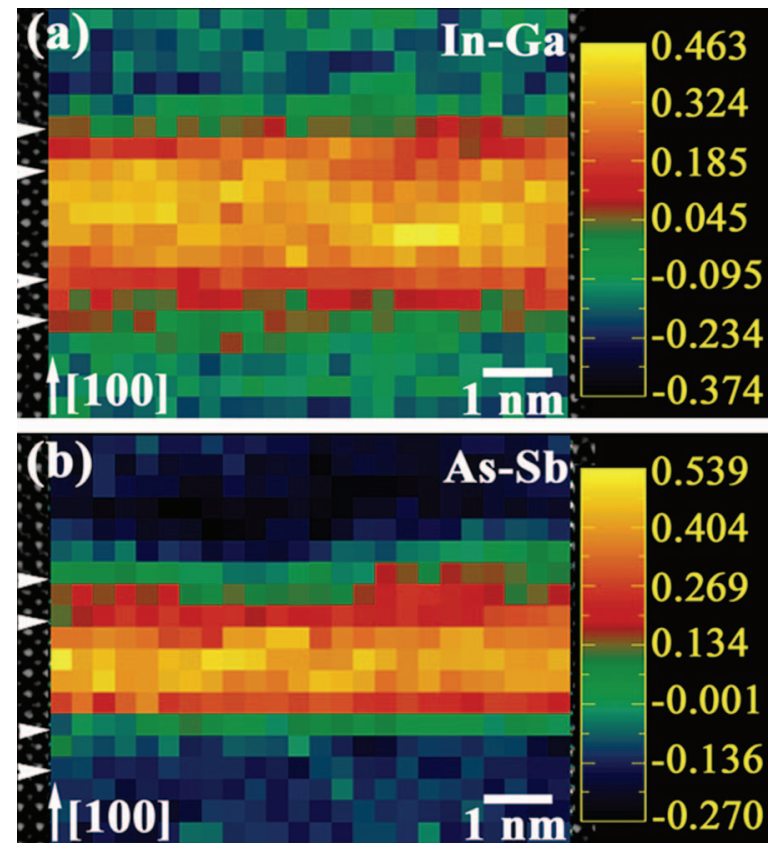

FIG. 3. (Color) Chemical maps of the phase image in Fig. 2(a), showing the variation of $C_{1}$ within (a) the In-Ga and (b) the As-Sb sublattices. The arrows locate the interfacial regions in Fig. 2(a).

2(b) and 2(c), respectively, where an asymmetry in grading at the interfacial regions is exhibited in both sublattices. Here, it is important to mention that similar analysis of a reconstructed phase image of an $\mathrm{AlGaAs} / \mathrm{GaAs}$ heterostructure (not shown) reveals grading in the phase profile along the $\mathrm{Al}-\mathrm{Ga}$ columns across each interface. However, the profiles along the adjacent rows of As columns were nearly constant, indicating that the line profiles depicted in Figs. 2(b) and 2(c) indeed represent changes in the $\mathrm{In}-\mathrm{Ga}$ and $\mathrm{As}-\mathrm{Sb}$ contents across interfacial regions.

To quantify changes in stoichiometry across each interface in Fig. 2(a), we implement a procedure based on FAC, which permits an independent analysis of the cation and anion sublattices. Detailed descriptions of the FAC method and its application to HRTEM image analysis are available elsewhere. ${ }^{10,13,14}$ It is sufficient to mention that applying the FAC procedure to a set of atomic site images [see inset in Fig. 2(a)] with different compositions and thickness yields a coefficient, $C_{1}$, for each image, which depicts the dominant variation in the set. Applying the FAC procedure to simulated phase images of $\mathrm{Al}_{x} \mathrm{Ga}_{(1-x)}$ As alloys shows that, for specimen thicknesses under $9 \mathrm{~nm}$, the dependence of $C_{1}$ on composition is linear, provided the images analyzed are all of constant thickness. ${ }^{17}$ Furthermore, this dependence is well described by the same linear equation independent of the magnitude of specimen thickness. To exploit this behavior the FAC procedure is performed in succession one [100] row at a time [e.g., all sites along line "a-b" in Fig. 2(a)], so that the effect of thickness variation between adjacent rows along [010] (in present case, the wedge direction of the sample) is excluded. Also, the cation and anion rows are analyzed independently, yielding separate maps of the variation in $C_{1}$ within the two sublattices. The validity of the proposed approach was confirmed by its application to simulated HRTEM images of AlGaAs/GaAs and InAs/InGaSb model structures with abrupt and graded interfaces. ${ }^{18,19}$

Figures 3(a) and 3(b) are, respectively, the results ob- 


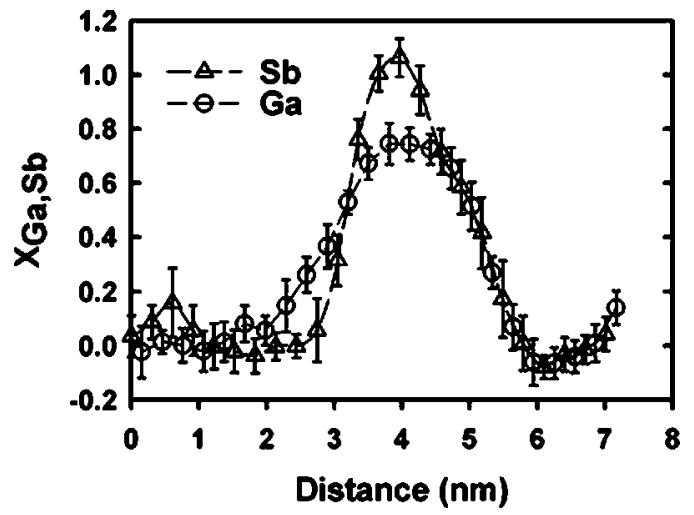

FIG. 4. Chemical profiles showing the mean compositions, $x_{\mathrm{Ga}}$ and $x_{\mathrm{Sb}}$, of each atomic layer along the [100] direction in Fig. 2(a).

tained for the In-Ga and the As-Sb sublattices. Distinct changes in $C_{1}$, indicating corresponding changes in composition, are clearly observed across interfaces in both sublattice images. In particular, the cation sublattice of the InGaSb-on-InAs interface (bottom) exhibits significant intermixing between In-Ga, whereas its anion sublattice is compositionally abrupt with no evidence for intermixing between As-Sb, thereby revealing that this interface is "InGaAs-like." On the other hand, the InAs-on-InGaSb interface (top) exhibits intermixing in both sublattices, indicating that it is "InGaAsSb-like". In addition, the As-Sb sublattice image of this interface indicates the presence of atomic scale roughness, which has also been observed in cross-sectional scanning tunneling microscope studies on InAs/GaSb heterostructures. ${ }^{20}$ These observations are consistent with the presence of a Ga-into-In exchange at the InGaSb-on-InAs interface and an As-into-Sb exchange at the InAs-on-InGaSb interface reported in III-V semiconductor systems. $^{21}$

The compositions, $x_{\mathrm{Ga}}$ and $x_{\mathrm{Sb}}$, of the individual atomic columns within the interfacial regions in Figs. 3(a) and 3(b) were determined from $C_{1}$ assuming the nominal compositions for the InAs and InGaSb regions far away from the interfaces. Based on the average values of $C_{1}$ in these regions and their standard deviations, the statistical error was estimated to be 0.07 for $x_{\mathrm{Ga}}$ and 0.08 for $x_{\mathrm{Sb}} \cdot{ }^{9}$ Figure 4 shows the $\mathrm{In}-\mathrm{Ga}$ and $\mathrm{As}-\mathrm{Sb}$ composition profiles normal to the interfaces, obtained by averaging along rows of atom sites parallel to the interface. The error bars denote standard deviations obtained for the corresponding row of atomic sites. The profiles clearly reveal the differences in characteristics of compositional change within the In-Ga and As-Sb sublattices. The negatively skewed In-Ga profile and the comparatively sharply peaked and positively skewed As-Sb profile relate to the differences in interface morphologies observed in the respective sublattice images shown in Figs. 3(a) and 3(b).
In summary, we have demonstrated that EPWF retrieval from HRTEM images enables atomic resolution compositional mapping of quaternary III-V semiconductor interfaces with intermixing in both cation and anion sublattices. Using this approach, we have independently quantified changes in the $\mathrm{In}-\mathrm{Ga}$ and $\mathrm{As}-\mathrm{Sb}$ contents across interfaces in an InAs $/ \mathrm{In}_{x} \mathrm{Ga}_{(1-x)} \mathrm{Sb}$ heterostructure, which revealed differences between the intermixing behavior within In-Ga and $\mathrm{As}-\mathrm{Sb}$ sublattices of the individual interfaces.

This research was sponsored by the Materials and Manufacturing Directorate, Air Force Research Laboratory, Wright-Patterson AFB, under Air Force Contract No. F33615-03-D-5801. The HRTEM experiments performed at NCEM, Berkeley, CA, was partly supported by the Director, Office of Science, Office of Basic Energy Science, U.S. Department of Energy under Contract No. DE-AC0376SF00098.

${ }^{1}$ For a recent review, see H. Kroemer, Physica E (Amsterdam) 20, 196 (2004).

${ }^{2}$ M. S. Daly, D. M. Symons, M. Lakrimi, R. J. Nicholas, N. J. Mason, and P. J. Walker, Semicond. Sci. Technol. 11, 823 (1996).

${ }^{3}$ N. Herres, F. Fuchs, J. Schmitz, K. M. Pavlov, J. Wagner, J. D. Ralston, P. Koidl, and G. Scmarcio, Phys. Rev. B 53, 15688 (1996).

${ }^{4}$ J. Spitzer, A. Hopner, M. Kuebal, M. Caradona, B. Jenichen, H. Neuroth, B. Brar, and H. Kroemer, J. Appl. Phys. 77, 811 (1995).

${ }^{5}$ Y. Wei, J. Bae, A. Gin, A. Hood, M. Razeghi, G. J. Brown, and M. Tidrow, J. Appl. Phys. 94, 4720 (2003).

${ }^{6}$ F. Fuchs, L. Burkle, R. Hamid, N. Herres, W. Pletschen, R. E. Sah, R. Kiefer, and J. Schmitz, Proc. SPIE 4288, 171 (2001).

${ }^{7}$ M. E. Twigg, B. R. Benett, P. M. Thibaldo, and B. V. Shannabrook, Appl. Phys. Lett. 67, 1609 (1995).

${ }^{8}$ A. Ourmazd, D. W. Taylor, J. Cunningham, and C. W. Tu, Phys. Rev. Lett. 34, 237 (1990).

${ }^{9}$ S. Thoma and H. Cerva, Ultramicroscopy 38, 265 (1991).

${ }^{10}$ J. L. Rouvière and N. Bonnet, Inst. Phys. Conf. Ser. 134, 11 (1993).

${ }^{11}$ W. M. J. Coene, A. Thust, M. Op de Beeck, and D. Van Dyck, Ultramicroscopy 64, 167 (1996).

${ }^{12}$ A. Thust and R. Rosenfield, in Proceedings of the International Congress on Electron Microscopy, edited by Hector A. Calderon and Miguel Jose Yacaman (Institute of Physics, Philadelphia, PA, 1998), Vol. 1, p. 119.

${ }^{13}$ P. Trebbia and N. Bonnet, Ultramicroscopy 34, 165 (1990).

${ }^{14}$ J. F. Aebersold, P. A. Stadelman, and J. L. Rouvière, Ultramicroscopy 62 , 171 (1996).

${ }^{15}$ C. Kisielowski, C. J. D. Hetherington, Y. C. Wang, R. Kilaas, M. A. O'Keefe, and A. Thust, Ultramicroscopy 89, 243 (2001).

${ }^{16}$ R. Kilaas, J. Microsc. 55, 181 (1997).

${ }^{17}$ K. Mahalingam, K. G. Eyink, G. J. Brown, and D. L. Dorsey, Int. J. Nanosci. 3, 723 (2004).

${ }^{18} \mathrm{~K}$. Mahalingam (unpublished).

${ }^{19}$ These simulations showed that the effect of small monotonic change in thickness along [100] [Fig. 2(a)] could also be estimated by a linear fit to the averaged values of $C_{1}$ in the two InAs layers, thereby retaining only the effects due to fine scale specimen roughness.

${ }^{20}$ R. M. Feenstra, D. A. Collins, Z.-Y. Ting, M. W. Wang, and T. C. McGill, Phys. Rev. Lett. 72, 2749 (1994).

${ }^{21}$ J. Steinshnider, J. Harper, M. Weimer, C.-H. Lin, and S. S. Pei, Phys. Rev. Lett. 85, 4562 (2000), and references therein. 